\title{
Anti-infectious Activity of Tryptophan Metabolites in the L-Tryptophan- L-Kynurenine Pathway
}

\author{
Koji Narui, ${ }^{a}$ Norihisa Noguchi, ${ }^{*}, a$ Aya Saito, ${ }^{b}$ Kazuhiro KaKimi,${ }^{c}$ Noboru Motomura, ${ }^{b}$ Kinya Kubo, ${ }^{d}$ \\ Shinichi TAKAMOTO, ${ }^{b}$ and Masanori SASATSU ${ }^{a}$ \\ ${ }^{a}$ Department of Microbiology, School of Pharmacy, Tokyo University of Pharmacy and Life Sciences; 1432-1 Horinouchi, \\ Hachioji, Tokyo 192-0392, Japan: ${ }^{b}$ Department of Cardiothoracic Surgery, Graduate School of Medicine, The University \\ of Tokyo; ${ }^{c}$ Department of Immunotherapeutics (Medinet), Graduate School of Medicine. The University of Tokyo; 7-3-1 \\ Hongo, Bunkyo-ku, Tokyo 113-8655, Japan: and ${ }^{d}$ Discovery Research Laboratories, Research Division, Kirin Pharma \\ Company, Limited; 3 Miyahara, Takasaki, Gunma 370-1295, Japan.
}

Received July 31, 2008; accepted October 23, 2008; published online October 30, 2008

To study the anti-infectious effect of a vascular allograft, the antimicrobial activity of tryptophan metabolites mediated by indoleamine 2,3-dioxygenase was determined. The growth of methicillin-resistant Staphylococcus aureus (MRSA) over $10 \mathrm{~h}$ in extracts from post-transplantation vascular allograft was significantly slower than that of extracts from non-transplantation vascular allograft regardless of the presence of tryptophan $(p<\mathbf{0 . 0 5})$. When the antimicrobial activity of the tryptophan metabolites in the L-tryptophan-L-kynurenine pathway was examined, 3-hydroxy-DL-kynurenine and $\alpha$-picolinic acid had strong antibacterial activity against MRSA, S. epidermidis, Escherichia coli, and multidrug-resistant Pseudomonas aeruginosa, although antimicrobial activities of anthranilic acid, 3-hydroxyanthranilic acid, and quinolinic acid against them were low. The results showed that, of the tested tryptophan metabolites, 3-hydroxy-DL-kynurenine and $\alpha$-picolinic acid contributed to the anti-infectious effects of the allograft by inhibiting of the growth of microorganisms.

Key words tryptophan metabolite; allograft; transplantation; methicillin-resistant Staphylococcus aureus; 3-hydroxy-DL-kynurenine

Infective endocarditis requires a combination therapy of surgery and long-term antibiotic administration. Surgery involves replacing an infected valve, with the three main replacement valve types being mechanical, bioprosthetic, and allograft heart valves. An allograft heart valve and a vascular allograft are tissue grafts donated from either cadaveric or brain dead donors. Allografts provide a better clinical outcome for infective cardiovascular disease than mechanical or stented bioprosthetic valves, and several clinical reports on the anti-infectious potential of allografts have been published. $^{1-6)}$

Indoleamine 2,3-dioxygenase (IDO), which is the rate-limiting enzyme in the L-tryptophan-L-kynurenine pathway, is induced by gamma interferon (IFN- $\gamma$ ). ${ }^{7,8)}$ Strong IDO activity leads to a nearly complete depletion of the essential amino acid tryptophan at the site of infection, which arrests the growth of several tryptophan-dependent microorganisms. $^{9-11)}$ The tryptophan metabolites act secondarily via an IFN- $\gamma$-mediated immunoregulated response in the anti-infection against the microorganism. ${ }^{12)}$ Therefore, it was predicted that anti-infections of the transplanted tissue graft were due to the depletion of tryptophan and the subsequent action of the tryptophan metabolites via an IFN- $\gamma$-mediated immunoregulated response. However, it was expected that tryptophan could be supplied anywhere; thus, there would be few chances for tryptophan to be depleted in vivo at the site of infection. Moreover, we have previously reported that the extract from the post-transplantation vascular allograft and 3hydroxy-DL-kynurenine, a tryptophan metabolite, had direct antibacterial activity against methicillin-resistant Staphylococcus aureus (MRSA) and that IDO and 3-hydroxy-DLkynurenine in the post-transplantation vascular allograft was produced greater than that in the non-transplantation vascular allograft. ${ }^{13)}$ To investigate the anti-infective mechanism of a vascular allograft in this study, we examined MRSA antibacterial activity of extracts from the vascular allografts in medium with or without tryptophan. In addition to MRSA, $S$. epidermidis, vancomycin-resistant Enterococcus faecalis (VRE), Escherichia (E.) coli, multidrug-resistant Pseudomonas aeruginosa (MDRP), and Candida (C.) albicans become pathogens after transplantation. ${ }^{14-19)}$ We examined the direct antimicrobial activities of six tryptophan metabolites against these microorganisms.

\section{MATERIALS AND METHODS}

Strains, Tryptophan Metabolites, and Medium MRSA N315 was used for assays to determine the antimicrobial activity of vascular grafts and the tryptophan metabolites. ${ }^{20)}$ S. epidermidis ATCC14990, VRE NCTC12201, E. coli ATCC11775, MDRP TP698 (clinical isolate), and C. albicans ATCC10231 were used for assays to determine the antimicrobial activity of the tryptophan metabolites. Tryptophan metabolites L-kynurenine, 3-hydroxy-DL-kynurenine, anthranilic acid, 3-hydroxyanthranilic acid, quinolinic acid, and $\alpha$-picolinic acid were purchased from Sigma-Aldrich (Tokyo, Japan). Mannitol-salt agar (MS agar; Eiken Chemical, Tokyo, Japan) was used to grow the MRSA, and heat-infusion agar (HI agar; Eiken Chemical, Tokyo, Japan) was used to grow the other microorganisms. Mueller-Hinton broth (MH broth; Oxoid, Hampshire, England) was used as the assays medium to determine the antimicrobial activity of vascular grafts and the tryptophan metabolites.

Antimicrobial Assay of Vascular Grafts The allografts were obtained from the experimental rat models as described previously. ${ }^{13)}$ Non- and post-transplantation vascular grafts were used as the IDO-negative graft and IDO-positive graft, respectively. The explanted grafts were transferred to $60 \mu \mathrm{l}$ 
of $2 \times \mathrm{MH}$ broth and incubated for $12 \mathrm{~h}$ at $35^{\circ} \mathrm{C}$ to extract the antibacterial agents from the grafts. After centrifugation at $15000 \mathrm{rpm}$ for $2 \mathrm{~min}$, the supernatant was collected and boiled for $2 \mathrm{~min}$. The supernatant collected was used as a $2 \times$ culture medium to determine the antibacterial activity of the grafts. An MRSA solution (approximately 5 colony forming units $(\mathrm{cfu}) / \mu \mathrm{l})$ and distilled water $(40 \mu \mathrm{l})$ with or without tryptophan (final concentration: $1 \mathrm{~mm}$ ) (Wako Pure Chemical Industries, Ltd., Osaka, Japan) were added into the $2 \times$ concentration culture medium $(50 \mu \mathrm{l})$. The mixture $(100 \mu \mathrm{l})$ was incubated at $35^{\circ} \mathrm{C}$ as well as antimicrobial susceptibility test. $^{21)}$ Five microliters of the culture were transferred every hour into $95 \mu \mathrm{l}$ of $\mathrm{MH}$ broth. Then, $50 \mu \mathrm{l}$ of the diluted culture were immediately spread onto MS agar. After incubation at $35^{\circ} \mathrm{C}$ for $24 \mathrm{~h}$, the plated colonies were counted.

Antimicrobial Assay of the Tryptophan Metabolites The antimicrobial activity of tryptophan metabolites, mediated by IDO against MRSA, was determined by the measurement of the minimum inhibitory concentration (MIC) using the microdilution method as outlined by Clinical and Laboratory Standards Institute. ${ }^{21)}$

An MRSA solution [approximately $5 \mathrm{cfu} / \mu \mathrm{l}], S$. epidermidis solution (approximately $1.5 \mathrm{cfu} / \mu \mathrm{l}$ ), VRE solution (approximately $30 \mathrm{cfu} / \mu \mathrm{l}$ ), E. coli solution (approximately $100 \mathrm{cfu} / \mu \mathrm{l}$ ), MDRP solution (approximately $50 \mathrm{cfu} / \mu \mathrm{l}$ ), or $C$. albicans solution (approximately $5 \mathrm{cfu} / \mu \mathrm{l}$ ) or distilled water $(40 \mu \mathrm{l})$ with or without the tryptophan metabolites were added into the $2 \times \mathrm{MH}$ broth. The mixture $(100 \mu \mathrm{l})$ was then incubated at $35^{\circ} \mathrm{C}$. Five microliters of the culture were transferred every hour into $95 \mu \mathrm{l}$ of $\mathrm{MH}$ broth. Then, $50 \mu \mathrm{l}$ of the diluted culture were immediately spread onto MS or HI agar. After incubation for $12 \mathrm{~h}$ at $35^{\circ} \mathrm{C}$, the colonies on the plate were counted. Antimicrobial activity was determined by the $\mathrm{IC}_{50}$ as described previously. ${ }^{22-24)}$ The $\mathrm{IC}_{50}$ for MRSA, $S$. epidermidis, and VRE were calculated from number of colonies at $8 \mathrm{~h}$ and for $E$. coli, MDRP, and C. albicans at 10 or $20 \mathrm{~h}$. Growth rate (percentage) at each level of tryptophan metabolite was measured in the values divided by the control which lacked tryptophan metabolite. The growth percentages and tryptophan metabolite concentrations were plotted on certain scales involving the special properties of the normal distribution curve. The $\mathrm{IC}_{50}$ was read off from the scale.

Statistical Analysis Differences in antimicrobial activity against the in vitro growth of MRSA for $10 \mathrm{~h}$ between the IDO-positive grafts and the IDO-negative graft were analyzed by a Student's $t$-test. Analyses were performed using SAS software JMP 5.01. For all comparisons, a $p$ value of $<0.05$ was considered to be statistically significant.

\section{RESULTS}

Antibacterial Assay of Vascular Grafts The IDO expression of the post-transplantation vascular graft (IDO-positive graft) was almost 60 -fold greater than that of the nontransplantation vascular graft (IDO-negative graft) (data not shown). The antibacterial activities of the IDO-positive grafts and the IDO-negative graft extracts were examined against MRSA (Fig. 1). MRSA growth curves in the extracts from those grafts are shown in Fig. 1A. MRSA growth in the extracts from the IDO-positive grafts at $10 \mathrm{~h}$ was significantly slower than that of the IDO-negative graft regardless of the
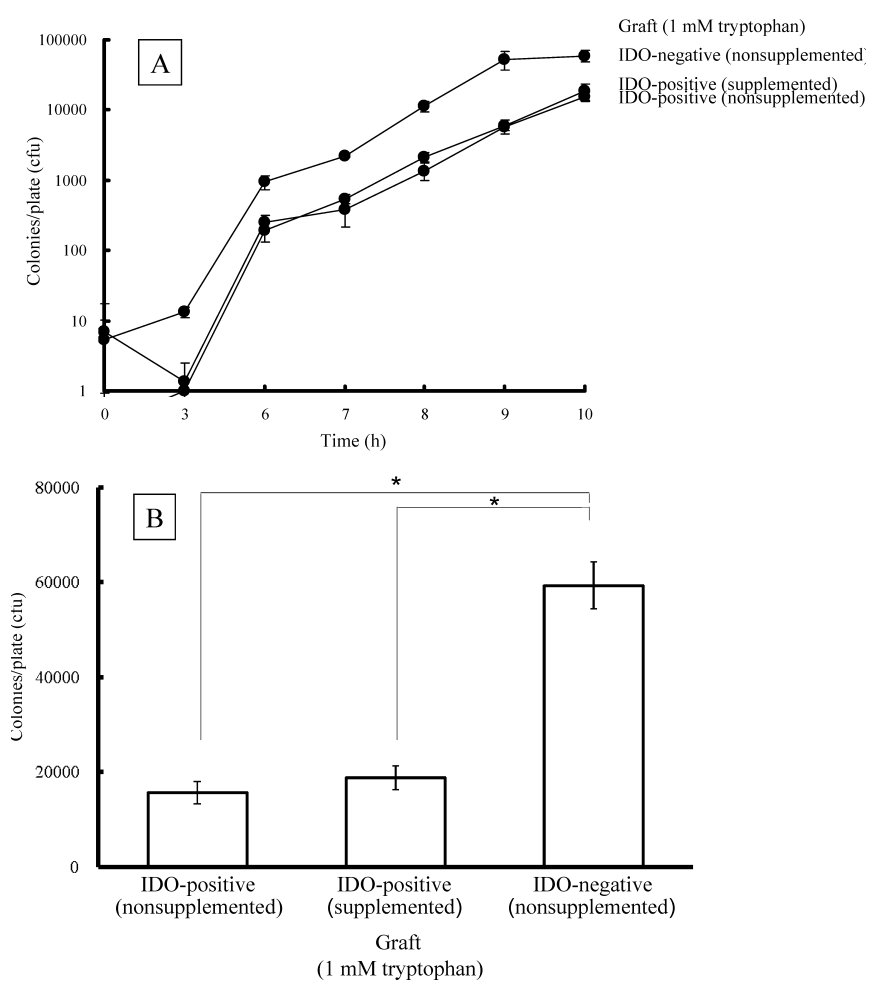

Fig. 1. Antimicrobial Activity of the IDO-Positive Graft and the IDONegative Graft Extracts against MRSA in Vitro

Non- and post-transplantation vascular grafts were used as the IDO-negative graft and the IDO-positive graft, respectively. Extracts from the IDO-positive grafts with or without tryptophan (final concentration: $1 \mathrm{~mm}$ ) were used. (A) MRSA growth curves in extracts from the IDO-negative grafts and the IDO-positive graft. (B) Growth inhibitory effect of the IDO-negative graft and the IDO-positive graft extracts on MRSA at $10 \mathrm{~h}$. Data represent the mean \pm S.D. of three experiments. $* p$ value $<0.05$ versus IDO-negative graft.

presence of tryptophan ( $p$ value $<0.05$ versus control) (Fig. 1B). These results show the presence of heat-stable antibacterial agent(s) in the IDO-positive grafts. In addition, the production of the antibacterial agent(s) was related to the expression of IDO. ${ }^{13)}$ Thus, these observations suggest that the tryptophan metabolite(s) are associated with the antibacterial activity of the allograft.

Antimicrobial Assay of the Tryptophan Metabolites MICs of the tryptophan metabolites, including L-kynurenine, 3-hydroxy-DL-kynurenine, anthranilic acid, 3-hydroxyanthranilic acid, quinolinic acid, and $\alpha$-picolinic acid for MRSA, were determined. The MIC for MRSA of 3-hydroxyDL-kynurenine was $256 \mu \mathrm{g} / \mathrm{ml}$ and the MICs of the other five metabolites were $\geq 1024 \mu \mathrm{g} / \mathrm{ml}$. These results indicated that the antibacterial activities of the tryptophan metabolites against MRSA could not be measured with the microdilution method in which the MIC is determined by the growth of bacteria after $24 \mathrm{~h}$. Therefore, to assess the antibacterial activity of the tryptophan metabolites, the growth of MRSA in $\mathrm{MH}$ broth that included a tryptophan metabolite was observed every hour (Fig. 2). L-Kynurenine did not inhibit the growth of MRSA. In contrast, 3-hydroxy-DL-kynurenine, anthranilic acid, 3-hydroxyanthranilic acid, quinolinic acid, and $\alpha$-picolinic acid dose-dependently inhibited MRSA growth. Furthermore, the growth of six microorganisms was measured in the presence of the metabolites and the $\mathrm{IC}_{50}$ was calculated to compare the antibacterial activities of the tryptophan metabolites against MRSA, S. epidermidis, VRE, E. 


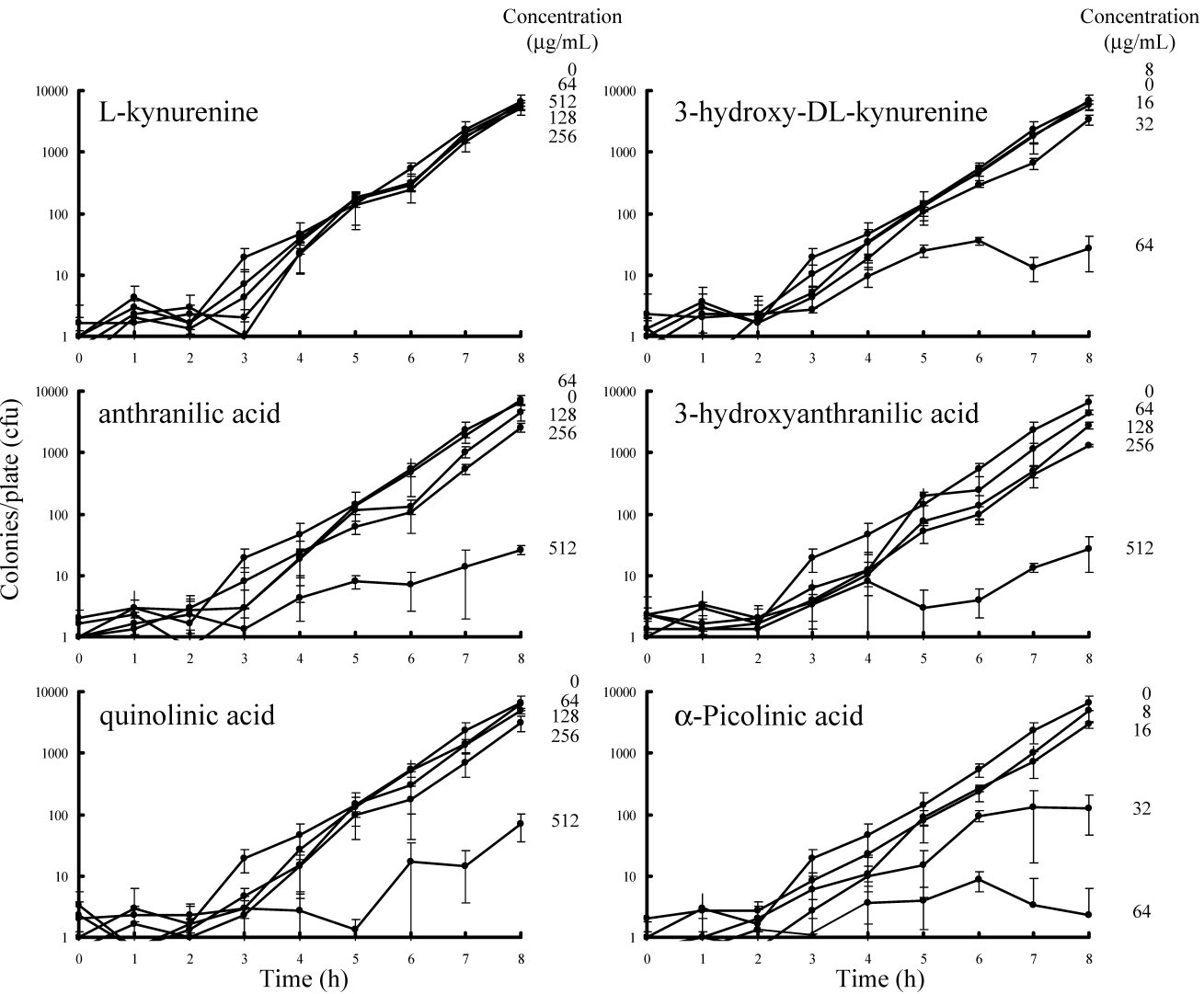

Fig. 2. MRSA Growth Curves with or without Tryptophan Metabolites at Various Concentrations

Data represent the mean \pm S.D. of three experiments.

Table 1. $\mathrm{IC}_{50} \mathrm{~s}$ of Tryptophan Metabolites against Different Microorganisms

\begin{tabular}{lcccccc}
\hline \hline \multirow{2}{*}{ Tryptophan metabolite } & \multicolumn{5}{c}{$\mathrm{IC}_{50}(\mu \mathrm{g} / \mathrm{ml})$ against the following microorganisms } \\
\cline { 2 - 7 } & MRSA & S. epidermidis & VRE & E. coli & MDRP & C. albicans \\
\hline L-Kynurenine & $\geq 1024$ & $\geq 1024$ & 336 & $\geq 1024$ & 371.2 & $\geq 1024$ \\
3-Hydroxy-DL-kynurenine & 31.2 & 39.2 & 57.6 & 24 & 25.6 & 137.6 \\
Anthranilic acid & 201.6 & 275.2 & 169.6 & 102.4 & 105.6 & $\geq 1024$ \\
3-Hydroxyanthranilic acid & 115.2 & 192 & 188.8 & 153.6 & 140.8 & $\geq 1024$ \\
Quinolinic acid & 227.2 & 384 & 240 & 71.6 & 51.2 & $\geq 1024$ \\
$\alpha$-Picolinic acid & 14 & 68.8 & 152 & & \\
\end{tabular}

$\mathrm{IC}_{50} ; 50 \%$ growth inhibitory concentration. MRSA, methicillin-resistant Staphylococcus aureus; S. epidermidis, Staphylococcus epidermidis; VRE, vancomycin-resistant Enterococcus faecalis; E. coli, Escherichia coli; MRDP, multidrug-resistant Pseudomonas aeruginosa; and C. albicans; Candida albicans.

coli, MDRP, and C. albicans, which cause infective endocarditis and infection after transplantation, (Table 1). LKynurenine had little antimicrobial activity against the tested microorganisms. 3-Hydroxy-DL-kynurenine had strong antibacterial activity for all tested bacteria, and no tryptophan metabolites had antifungal activity. 3-Hydroxy-DL-kynurenine, anthranilic acid, 3-hydroxyanthranilic acid, and quinolinic acid had higher antimicrobial activity against Gramnegative bacilli ( $E$. coli and $P$. aeruginosa) than for Grampositive cocci (MRSA, S. epidermidis and VRE). $\alpha$-Picolinic acid had strong antibacterial activity against all tested bacteria except VRE. The antibacterial activity of 3-hydroxy-DLkynurenine, 3-hydroxyanthranilic acid, quinolinic acid, and $\alpha$-picolinic acid against MRSA were highest in Gram-positive cocci (MRSA, S. epidermidis, and VRE). In contrast, the antibacterial activity of the tryptophan metabolite against MDRP was almost equal to its activity against $E$. coli.

\section{DISCUSSION}

Since the 1990s, several clinical reports have been published on the anti-infectious potential of allografts. ${ }^{1-6)}$ Depleting the essential amino acid tryptophan by IDO arrests the growth of several tryptophan-dependent microorganisms. ${ }^{9-11)}$ However, our antimicrobial activity assays clearly showed remarkable MRSA growth inhibition in supernatant extracted from the IDO-positive graft in the presence of tryptophan. To our knowledge, our report is the first to prove that the anti-infective activity of the L-tryptophan-L-kynurenine pathway is not due to tryptophan depletion.

The antibacterial activity of tryptophan metabolites against MRSA was not demonstrated by determining MICs. Rather, the growth and the $\mathrm{IC}_{50}$ of the metabolites against the six microorganisms tested in this study were measured in medium with or without the tryptophan metabolites. 3-Hydroxy-DL- 
kynurenine and $\alpha$-picolinic acid had higher antibacterial activity against MRSA, $S$. epidermidis, E. coli, and MDRP than L-kynurenine, anthranilic acid, 3-hydroxyanthranilic acid, and quinolinic acid. The notable feature of the antibacterial activity is the effect against drug resistant bacteria such as MRSA and MDRP, although the antibacterial mechanism is unknown. The $\mathrm{IC}_{50}$ of tryptophan metabolites against microorganisms were high, although $\alpha$-picolinic acid showed the lowest $\mathrm{IC}_{50}(14 \mu \mathrm{g} / \mathrm{ml})$ against MRSA, considering the breakpoint of vancomycin, anti-MRSA agent, is $2 \mu \mathrm{g} / \mathrm{ml} .^{25)} \mathrm{In}$ addition, the 3-hydroxy-DL-kynurenine of $15.7 \pm 5.11 \mathrm{ng} / \mathrm{mg}$ was included in IDO-positive graft. ${ }^{13)}$ The concentration of the 3-hydroxy-DL-kynurenine in IDO-positive graft is very low compared with $\mathrm{IC}_{50}$ of it. These results suggest that the antibacterial activity of tryptophan metabolites can be a part of that of IDO-positive graft and tryptophan metabolites can synergistically act against microorganisms. In fact, multiple tryptophan metabolites had antibacterial activity and showed different antimicrobial spectra.

This study showed that 3 -hydroxy-DL-kynurenine and $\alpha$ picolinic acid contribute to the anti-infectious activity of allografts by direct growth inhibition of microorganisms. Antimicrobial mechanism of 3-hydroxy-DL-kynurenine is not published. In contrast, this is consistent with the finding by Cai et al. that depriving free nutrient iron by $\alpha$-picolinic acid, a cheap and safe chelating agent, is attributable to its direct antimicrobial activity. ${ }^{26)}$ Based on this study, there is a possibility that infectious disease could be prevented and treated in a new and unique way. Administering a tryptophan metabolite(s) and/or inducing IDO could provide direct antimicrobial activity and may even be applicable in cases of infectious diseases caused by drug resistant microorganisms.

Acknowledgements The authors wish to thank S. Matsumoto, S. Naito, T. Suzuki, Y. Ando, T. Matsumura, and M. Wakabayashi for their expert technical assistance. This work was supported by grants for private universities provided by the Ministry of Education, Culture, Sports, Science and Technology and the Promotion and Mutual Aid Corporation for Private Schools of Japan.

\section{REFERENCES}

1) Tuna I. C., Orszulak T. A., Schaff H. V., Danielson G. K., Ann. Thorac. Surg., 49, 619-624 (1990).

2) Sabik J. F., Lytle B. W., Blackstone E. H., Marullo A. G., Pettersson G. B., Cosgrove D. M., Ann. Thorac. Surg., 74, 650-659 (2002).

3) Yankah A. C., Pasic M., Klose H., Siniawski H., Weng Y., Hetzer R., Eur. J. Cardiothorac. Surg., 28, 69-75 (2005).
4) Grinda J. M., Mainardi J. L., D’Attellis N., Bricourt M. O., Berrebi A., Fabiani J. N., Deloche A., Ann. Thorac. Surg., 79, 767-771 (2005).

5) Vogt P. R., von Segesser L. K., Jenni R., Niederhauser U., Genoni M., Kunzli A., Schneider J., Turina M. I., Eur. J. Cardiothorac. Surg., 11, 53-61 (1997).

6) Haydock D., Barratt-Boyes B., Macedo T., Kirklin J. W., Blackstone E., J. Thorac. Cardiovasc. Surg., 103, 130-139 (1992).

7) Mellor A. L., Munn D. H., Nat. Rev. Immunol., 4, $762-774$ (2004).

8) Munn D. H., Zhou M., Attwood J. T., Bondarev I., Conway S. J., Marshall B., Brown C., Mellor A. L., Science, 281, 1191-1193 (1998).

9) Dai W., Pan H., Kwok O., Dubey J. P., J. Interferon Res., 14, 313-317 (1994).

10) Murray H. W., Szuro-Sudol A., Wellner D., Oca M. J., Granger A. M., Libby D. M., Rothermel C. D., Rubin B. Y., Infect. Immun., 57, 845849 (1989).

11) Hucke C., MacKenzie C. R., Adjogble K. D., Takikawa O., Daubener W., Infect. Immun., 72, 2723-2730 (2004).

12) Mucci A., Varesio L., Neglia R., Colombari B., Pastorino S., Blasi E., Med. Microbiol. Immunol., 192, 71-78 (2003).

13) Saito A., Motomura N., Kakimi K., Narui K., Noguchi N., Sasatsu M., Kubo K., Koezuka Y., Takai D., Ueha S., Takamoto S., J. Thorac. Cardiovasc. Surg., 136, 159-167 (2008).

14) Schwabe M., Juttner E., Blaich A., Potthoff K., Pisarski P., Waller C. F., Transpl. Infect. Dis., 9, 46-50 (2007).

15) El-Khoury J., Fishman J. A., Transpl. Infect. Dis., 5, 121-125 (2003).

16) Domenig C., Traunmuller F., Kozek S., Wisser W., Klepetko W., Steininger R., Spiss C., Thalhammer F., Transplantation, 71, 744 745 (2001).

17) Laouad I., Buchler M., Noel C., Sadek T., Maazouz H., Westeel P. F., Lebranchu Y., Transplant. Proc., 37, 2834-2836 (2005).

18) Rice J. C., Peng T., Kuo Y. F., Pendyala S., Simmons L., Boughton J., Ishihara K., Nowicki S., Nowicki B. J., Am. J. Transplant., 6, 23752383 (2006).

19) Cosgrove S. E., Sakoulas G., Perencevich E. N., Schwaber M. J., Karchmer A. W., Carmeli Y., Clin. Infect. Dis., 36, 53-59 (2003).

20) Kuroda M., Ohta T., Uchiyama I., Baba T., Yuzawa H., Kobayashi I., Cui L., Oguchi A., Aoki K., Nagai Y., Lian J., Ito T., Kanamori M., Matsumaru H., Maruyama A., Murakami H., Hosoyama A., MizutaniUi Y., Takahashi N. K., Sawano T., Inoue R., Kaito C., Sekimizu K., Hirakawa H., Kuhara S., Goto S., Yabuzaki J., Kanehisa M., Yamashita A., Oshima K., Furuya K., Yoshino C., Shiba T., Hattori M., Ogasawara N., Hayashi H., Hiramatsu K., Lancet, 357, 1225-1240 (2001).

21) Clinical and Laboratory Standards Institute, "Methods for Dilution Antimicrobial Susceptibility Tests for Bacteria That Grow Aerobically," approved standard-seventh edition, M7-A7, Clinical and Laboratory Standards Institute, Wayne, PA, 2006.

22) Treffers P. H., J. Bacteriol., 72, 108-114 (1956).

23) Narui K., Noguchi N., Wakasugi K., Sasatsu M., Biol. Pharm. Bull., 25, 1533-1536 (2002).

24) Noguchi N., Emura A., Sasatsu M., Kono M., Biol. Pharm. Bull., 17, $352-355$ (1994).

25) Clinical and Laboratory Standards Institute, "Performance Standards for Antimicrobial Susceptibility Testing," seventeenth informational supplement, Clinical and Laboratory Standards Institute, Wayne, PA, 2007.

26) Cai S., Sato K., Shimizu T., Yamabe T., Hiraki M., Sano C., Tomioka H., J. Antimicrob. Chemother., 57, 85-93 (2006). 